\title{
High resolution soft X-ray spectroscopy of M 87 with the reflection grating spectrometers on $X M M-$ Newton
}

\author{
I. Sakelliou ${ }^{1, \star}$, J. R. Peterson ${ }^{2}$, T. Tamura ${ }^{3}$, F. B. S. Paerels ${ }^{2}$, J. S. Kaastra ${ }^{3}$, E. Belsole ${ }^{4}$, H. Böhringer ${ }^{5}$, \\ D. M. Worrall ${ }^{9}$ \\ 1 Mullard Space Science Laboratory, University College London, Holmbury St. Mary, Dorking, \\ Surrey RH5 6NT, UK \\ 2 Columbia Astrophysics Laboratory, 550 W. 120th St., New York, NY10027, USA \\ 3 SRON National Institute for Space Research, Sorbonnelaan 2, 3584 CA Utrecht, The Netherlands \\ ${ }^{4}$ CEA Saclay, Service d'Astrophysique, 91191 Gif-sur-Yvette, France \\ 5 Max-Planck-Institut für Extraterrestrische Physik, Germany \\ 6 Department of Physics, University of California, Santa Barbara, CA 93106, USA \\ 7 NASA/GSFC, Code 662, Greenbelt, MD 20771, USA \\ ${ }^{8}$ NIS-2, MS D436, Los Alamos National Laboratory, USA \\ 9 Department of Physics, University of Bristol, Tyndall Avenue, Bristol BS8 1TL, UK
} G. Branduardi-Raymont ${ }^{1}$, C. Ferrigno ${ }^{3}$, J. W. den Herder $^{3}$, J. Kennea ${ }^{6}$, R. F. Mushotzky ${ }^{7}$, W. T. Vestrand ${ }^{8}$, and

Received 7 February 2002 / Accepted 12 June 2002

\begin{abstract}
We present high-resolution X-ray spectroscopic observations of M 87 with the Reflection Grating Spectrometers on XMM-Newton. We detect strong $\mathrm{K}$-shell line emission from $\mathrm{N}, \mathrm{O}, \mathrm{Ne}, \mathrm{Mg}$, some emission from He-like $\mathrm{Si}$, a fully resolved set of Fe L-shell emission spectra, and some emission from C. The angular intensity distributions of the strong emission lines are detectably resolved on scales (15-160)". The gas in the inner arcmin of M 87 has a multi-phase structure, as indicated by the similarity of the emission line profiles of $\mathrm{Fe} \mathrm{L}$ shell ions with widely separated ionization potentials. The global $\mathrm{Fe} \mathrm{L}$ spectrum is approximately consistent with an isothermal plasma at $k T_{\mathrm{e}} \sim 1.8 \mathrm{keV}$, in addition to a component with a temperature distribution appropriate to an isobaric cooling flow, but with a minimum temperature cutoff of $k T_{\min } \approx 600 \mathrm{eV}$. The behaviour of this cooling-flow component is qualitatively similar to what is seen in other cooling flow clusters. Finally, we do not find any strong evidence for a spatial variation in abundances due to resonance scattering redistribution in the inner arcminute of the core.
\end{abstract}

Key words. galaxies: clusters: general - galaxies: clusters: individual: virgo - galaxies: individual: M 87 - galaxies: cooling flows - galaxies: abundances - X-rays: galaxies

\section{Introduction}

The giant elliptical galaxy M 87, its active nucleus, and its halo have been the subject of intensive studies at all wavelengths (see e.g., Röser \& Meisenheimer 1999). The extended diffuse emission has been mapped in neutral atomic and molecular gas, and in the highly-ionised hot gas that is revealed through X-ray emission. X-ray observations with the Einstein Observatory first found evidence for a mass of rapidly cooling X-ray emitting gas at the core of the system. Estimates for the density and temperature of this gas suggested the presence of a "cooling flow" with a total mass deposition rate of $\sim 10 M_{\odot} \mathrm{yr}^{-1}$ (Fabricant et al. 1980; Stewart et al. 1984;

Send offprint requests to: I. Sakelliou,

e-mail: irini@star.sr.bham.ac.uk

* Present address: School of Physics \& Astronomy, University of Birmingham, Edgbaston, Birmingham B15 2TT, UK.
Fabian et al. 1984). Direct spectroscopic evidence for the presence of radiatively cooling gas was obtained with the Focal Plane Crystal Spectrometer on Einstein (Canizares et al. 1979) and the Solid State Spectrometer (Lea et al. 1982).

The advent of high-sensitivity X-ray imaging and spectroscopy with the Chandra and XMM-Newton Observatories now provides the opportunity for a detailed study of the physical conditions in the cooling gas and its interaction with its environment. High spatial resolution images of M 87 have been obtained with Chandra (Wilson \& Yang 2001). Medium resolution XMM-Newton spatially resolved spectroscopy has been published by Böhringer et al. (2001), Belsole et al. (2001), Molendi \& Pizzolato (2001) and Matsushita et al. (2001). For objects of moderate angular extent (up to approximately 1 arcmin), the Reflection Grating Spectrometers (RGS) on $X M M-N e w t o n$ have the unique capability to provide high resolution X-ray spectroscopy coupled with some spatial 
resolution in the cross-dispersion direction. This combination of capabilities is uniquely suited to conducting a sensitive study of the thermodynamic properties of the cooling gas. Here, we describe preliminary results of such a study, based on data obtained during the XMM-Newton Performance Verification phase.

\section{Data reduction and analysis}

M 87 was observed on June 19,2000 , for a total of $\approx 60 \mathrm{ksec}$. All instruments on board XMM-Newton were operating. For a description of the observatory and its instrumentation, we refer to Jansen et al. (2001). The RGS has been described by den Herder et al. (2001).

The data reduction was performed with the SAS (XMMNewton Science Analysis System), using the most recent calibration for wavelength scale and effective area. Data reduction was performed with RGSPROC-0.91; spectral response files were generated with RGSRMfGeN-0.34. Periods of high background were removed from the data, leaving a net effective exposure time of $\approx 40 \mathrm{ksec}$. The total count rate in the first spectral order is about 1.3 counts s$^{-1}$ over the 5-35 ̊ band (both spectrometers combined).

Spectra were extracted from both RGS images by selecting events that lie within a rectangular mask with a width in the cross dispersion direction of 30 pixels, or $1.1 \mathrm{arcmin}$. This angular width encompasses the visible bulk of the emission detected in the RGS. The background in the RGS images within this aperture is almost negligible (a few percent of the source signal). At the distance of M 87 (17 Mpc; Freedman et al. 1994), the $1.1 \mathrm{arcmin}$ width corresponds to $\sim 5.4 \mathrm{kpc}$, or a radius of $2.7 \mathrm{kpc}$. The spatial resolution of the RGS in the cross-dispersion direction is approximately $15 \mathrm{arcsec}$, so that our extraction region covers about four spatial resolution elements. Along the dispersion direction, the equivalent spatial resolution is about 20 arcsec. The bright core has a diameter of order 10 arcsec, and we expect all spectral lines to be slightly broadened: the broadening is $\sim 0.124 \times \theta$ (1st spectral order; XMM-Newton UHB), where $\theta$ is the source extent in arcmin. For comparison, the wavelength resolution and spectral resolving power of the spectrometers are $\sim 0.06 \AA$, and $\sim 250$ at $15 \AA(F W H M)$ respectively.

Of course, we also detect photons from all gas in those parts of the halo that are projected onto our aperture. Due to the much larger extent of the outer halo, it appears as a near-isotropic component in the image, which means that halo photons appear as a pseudo-continuum in our spectra. The intensity of this pseudo-continuum can be suppressed by using the intrinsic energy resolution of the RGS focal plane CCD cameras, and filtering the photons by matching each photon's dispersion coordinate to its expected possible range of CCD pulse heights. The sensitivity of the spectrometer to remaining "out of band" photons is automatically included when we calculate the response of the RGS, based on the response to monochromatic light from an extended source of given angular distribution. We used the standard cut on CCD pulse heights, which includes $90 \%$ of the pulse height response to monochromatic radiation (about $\sim 200 \mathrm{eV}$ wide).
Figure 1 shows the first- and second- order spectra. As can be seen, we detect strong $\operatorname{Ly} \alpha$ emission from the hydrogenlike ions of $\mathrm{Mg}, \mathrm{Ne}, \mathrm{O}, \mathrm{N}$, and $\mathrm{C}$, in addition to emission from essentially all species in the Fe L series (Fe XVII, or Ne-like, through Fe XXIV, or Li-like). No significant He-like oxygen is detected (O VII resonance, inter-combination, and forbidden lines at 21.60, 21.80, and $22.10 \AA$ ).

\section{Thermal structure}

Spectroscopy in the 5-35 $\AA$ RGS range is particularly well suited to studying the mix in temperature and abundance of cool gas in the centre of M 87. The presence of all eight charge states of the Fe L series, with ionization potentials between $500 \mathrm{eV}$ (to create the Ne-like ion) and $2000 \mathrm{eV}$ will allow us to use iron as a thermometer, to probe the electron-temperature distribution between 200 and $2000 \mathrm{eV}$. There are no uncertainties associated with abundances, because the temperature distribution is constrained solely by the ionization balance of iron. Emission from the hydrogen-like ions of $\mathrm{Mg}, \mathrm{Ne}, \mathrm{O}$, and, to a lesser extent, $\mathrm{N}$ and $\mathrm{C}$, can arise from gas at temperatures outside the $\mathrm{Fe} \mathrm{L}$ range, and therefore some uncertainties may remain in the absolute abundances of these elements from the analysis of the RGS spectrum. Finally, because the core of M 87 is moderately resolved in the RGS on scales of order $15^{\prime \prime}$, the analysis should also provide constraints on the spatial temperature and abundance structure below $k T_{\mathrm{e}} \sim 2 \mathrm{keV}$. However, this coupled spatial-thermal structure of the core is complicated, and we therefore chose to restrict the discussion in this paper to a preliminary analysis of the data in terms of a simple spatial model, based on the directly observed image, convolved with a multi-temperature emission spectrum. A full characterisation of the RGS spectrum of an extended source would require a more detailed theoretical model for the spatial distribution of the extended source, and better knowledge of the instrument and sky backgrounds.

Technically, the spectral analysis proceeded as follows. A collisional-ionization equilibrium spectrum of chosen temperature distribution and chosen abundance pattern was convolved with a model that describes the spatial distribution of the emitting source. This spatial model was derived by using the $X M M$-Newton MOS image in the relevant pulse height range $[(0.5-2.5) \mathrm{keV}]$, properly scaled and collapsed onto the RGS dispersion direction. This spatial model was convolved with the model emission spectrum, and compared to the data. Only the wavelength range between 8 and $35 \AA$ was used, since at the time of writing the calibration accuracy below $8 \AA$ was poorer, and therefore a $8 \AA$ low limit was a safe approach. The flux normalisation of an extended source in this procedure is such that it reproduces the counts detected within the spatial extraction region. Therefore the numbers we quote have not been corrected for the photons outside the extraction region. Emission from the active nucleus, taken to be a point source, was modelled with a power-law spectrum whose parameters are based on our analysis of the EPIC spectrum (photon index $\Gamma=2.3$, normalisation $7.9 \times 10^{49}$ photons $\mathrm{s}^{-1} \mathrm{keV}^{-1}$ at $1 \mathrm{keV}$ ). Foreground absorption by our own Galaxy was modelled using a column density $N_{\mathrm{H} \text {, Gal }}=2.5 \times 10^{20} \mathrm{~cm}^{-2}$. We also allowed 


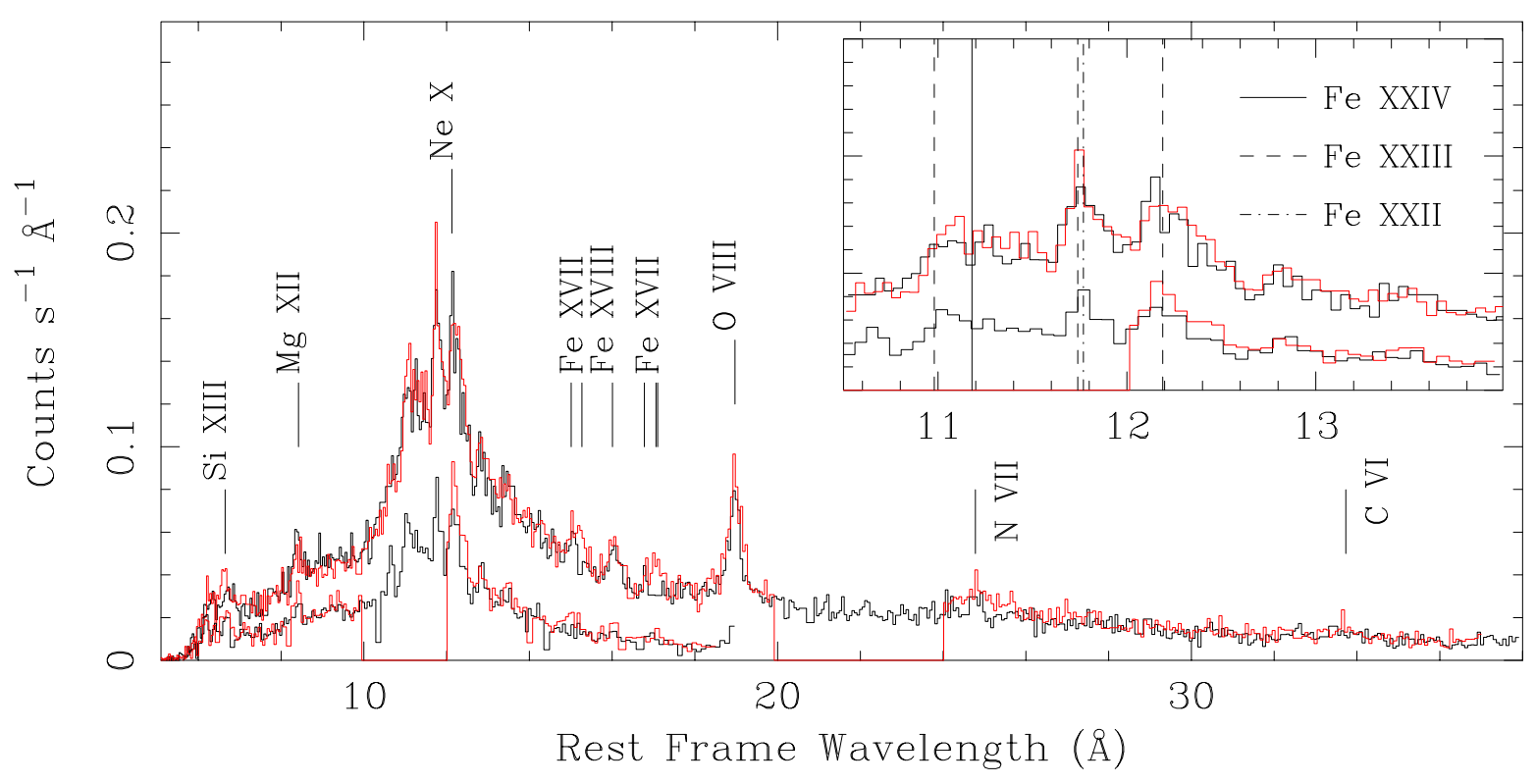

Fig. 1. The total RGS spectrum of the central 1.1 arcmin of M 87, in the source rest frame. The spectra from the two separate RGS instruments have been superimposed (RGS1 in black, RGS2 in red). We also show the corresponding second order spectra (whose full range covers the 2.5-17.5 $\AA$ band). First and second order spectra have similar wavelength resolution, due to the fact that the source is spatially resolved. Background has not been removed. The positions of $\operatorname{Ly} \alpha$ lines has been marked, as well as those of the strongest lines of the two lowestionization members of the Fe L series. The inset shows the 10.5-14 $\AA$ region on an expanded scale, with the positions of the strongest lines from the three highest-ionization members of the Fe L series.

for intrinsic absorption at $\mathrm{M} 87$, modelled with a neutral col-

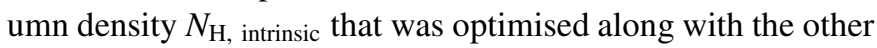
free parameters, and fixed covering factor 0.5. A small amount of photoelectric absorption by $\mathrm{O}$ intrinsic to the spectrometers was modelled as absorption by a pure oxygen column density of $N_{\mathrm{O}}=3 \times 10^{17} \mathrm{~cm}^{-2}$, based on observations of bright, unabsorbed extragalactic continuum sources.

A background spectrum was extracted from RGS data of blank sky (revolution70). The background RGS spectrum was extracted in a similar way as the source spectrum, and scaled properly to match the RGS observation of M 87. The contribution from the background is relatively low as shown in Fig. 2.

Calculation of the model emission spectra and convolution with the spatial distribution model were carried out using the SPEX package (Kaastra et al. 1996). The assumption that we can model the spatial distribution by convolving the emission spectrum due to an on-axis point source with a suitably scaled angular distribution (instead of properly taking the wavelength dependence of the spatial response into account) is, in view of the compactness of the source, only a minor one, and does not limit or affect our conclusions.

The simultaneous appearance of widely separated (in ionization potential) $\mathrm{Fe} \mathrm{L}$ charge states already indicates that the core is not isothermal; moreover, the shape of the emission features from different ions suggests similar spatial profiles for different temperature components. We therefore first attempted a model that naturally allows for a multi-temperature and multi-phase structure, the isobaric cooling-flow (ICF) prescription (Johnstone et al. 1992). Gas at sub-keV temperatures, and at densities of $\gtrsim 10^{-3} \mathrm{~cm}^{-3}$, has to radiatively cool rapidly, and would not be able to avoid cooling to low temperatures if radiative cooling was the only term in the thermal balance. However, spectroscopic observations of essentially all cooling flows indicate that there is a very significant lack of gas at low temperatures (Kaastra et al. 2001; Peterson et al. 2001; Tamura et al. 2001). Adapting the procedure followed by the above mentioned work on cooling-flow spectroscopy, we introduce a minimum temperature to the isobaric cooling-flow temperature distribution, in order to avoid overproduction of line emission in the lower few Fe L ions in a cooling flow that correctly reproduces the emission from the higher charge states.

In agreement with the CCD spectral analysis (Finoguenov et al. 2001), to this cooling flow we add a single temperature component (1T) to account for emission from the hotter regions surrounding the core, merging into the hot, extended intracluster medium. The abundances of $\mathrm{C}, \mathrm{N}, \mathrm{O}, \mathrm{Ne}, \mathrm{Mg}$, and $\mathrm{Fe}$ were left free, but tied to be the same in both components. The abundances of all other elements were set to their solar values relative to $\mathrm{Fe}$; we do not expect that their actual value effects the final results since the M 87 RGS spectrum [(8-35) $\AA$ ] is not sensitive to emission from elements such as $\mathrm{Ca}, \mathrm{Ar}, \mathrm{Al}, \mathrm{Si}$, and S. Solar abundances were taken from Anders \& Grevesse (1989). The best-fit parameters are given in Table 1.

This "1T+ICF" model reproduces the observed spectrum reasonably well, as is evident from Fig. 2. An inspection of the relative contributions of the isothermal component and the cooling flow component to the spectrum (ICF:1T 1:4) reveals that it is actually dominated by the isothermal component; the main contribution of the cooling flow are the faint $\mathrm{Fe}$ L lines between 12 and $16 \AA$. As stated above, emission from $\mathrm{H}$-like ions, especially those of $\mathrm{Mg}, \mathrm{Ne}$, and $\mathrm{O}$, can also be produced by even hotter gas, which is not included in our model. 

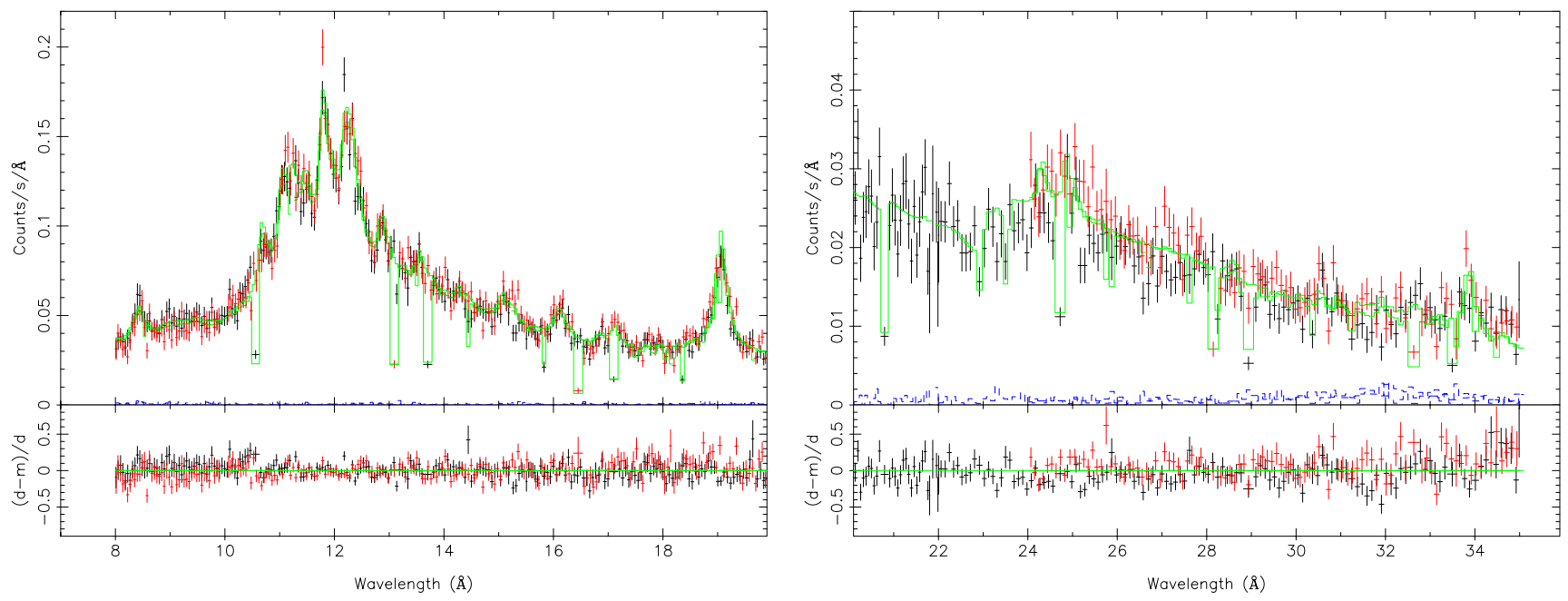

Fig. 2. The spectrum of the core of M 87 fit with the $1 T+I C F$ model, in the $8-20 \AA$ range (left), and the $20-36 \AA$ range (right). The RGS1 (black) and RGS2 (red) first order spectra were fitted simultaneously. The best fitting model spectrum is also shown (in green). The background spectra employed in the modelling are shown in blue. The bottom panels show the post-fit residuals. The large "dips" seen in both the data and the models correspond to CCD boundaries and/or dead columns.

Table 1. Fitting the $1 \mathrm{~T}+\mathrm{ICF}$ model to the core of M 87. Errors are statistical (90\% confidence level).

\begin{tabular}{lc}
\hline \hline parameter & value \\
\hline$N_{\mathrm{H}, \text { intrinsic }}$ & $6 \pm 2$ \\
covering factor $^{a}$ & 0.5 \\
$\dot{M}^{b}$ & $6 \pm 1$ \\
$T_{\min }(\mathrm{keV})$ & $0.6 \pm 0.02$ \\
$E M_{\mathrm{hot}}^{c}$ & 8.1 \\
$T_{\mathrm{hot}}(\mathrm{keV})$ & $1.8 \pm 0.05$ \\
$\mathrm{C}^{d}$ & $1.0 \pm 0.3$ \\
$\mathrm{~N}^{d}$ & $0.8 \pm 0.3$ \\
$\mathrm{O}^{d}$ & $0.49 \pm 0.04$ \\
$\mathrm{Ne}^{d}$ & $0.7 \pm 0.2$ \\
$\mathrm{Mg}^{d}$ & $0.9 \pm 0.2$ \\
$\mathrm{Fe}^{d}$ & $0.77 \pm 0.04$ \\
$\chi^{2} / v$ & $962 / 694$ \\
\hline
\end{tabular}

a "Intrinsic" column density in units of $10^{21} \mathrm{~cm}^{-2}$, and the covering factor.

${ }^{b}$ Mass deposition rate in units of $M_{\odot} \mathrm{yr}^{-1}$.

$c$ Volume emission measure in units of $10^{64} \mathrm{~cm}^{-3}$.

${ }^{d}$ Metal abundances relative to the solar values.

Evidence for hotter gas at larger radii comes from the analysis of the observations from imaging detectors (e.g., Matsushita et al. 2001). Therefore, the derived abundances of those elements have to be regarded with some skepticism. The estimate for the total mass deposition rate, $\dot{M}$, is strongly coupled to that for the minimum cooling flow temperature, for the simple reason that a high $\dot{M}$ over-predicts emission in the lowest Fe L ions, which has to be countered by a relatively high minimum temperature. It is worth noting that the previously morphologically determined deposition rate, $\dot{M}=39_{-9}^{+2} M_{\odot} \mathrm{yr}^{-1}$ (Peres et al. 1998), when scaled to our 1.1 arcmin aperture, predicts an $\dot{M} \sim 7 M_{\odot} \mathrm{yr}^{-1}$. Still, emission from cooling gas is not seen at temperatures below $k T_{\mathrm{e}} \sim 600 \mathrm{eV}$, and all difficulties with understanding this result in other cooling flow clusters apply equally to the present case of M 87. How strongly emission from cooling gas at the lower temperatures is ruled out is illustrated in Fig. 3, where we show the measured spectrum, overlaid with the " $1 \mathrm{~T}+\mathrm{ICF}$ " spectral model, but this time with the contribution of the cooling flow below $k T_{\mathrm{e}}=600 \mathrm{eV}$ included.

\section{The spatial distributions of temperature and abundance on larger scales}

Finally, we attempt to characterise the physical state of the line emitting gas on spatial scales comparable to, or somewhat larger than the spatial resolution of the RGS, by examining the intensity distribution in a number of diagnostic, strong emission lines. Figure 4 shows their cross-dispersion profiles, binned in bins of 15 arcsec in extent in the cross-dispersion direction, and accumulated over $0.2 \AA$ wide intervals in the spectral dimension. The contribution of the continuum to the profiles has been subtracted, by constructing cross-dispersion profiles in continuum windows, and interpolating the strength of the continuum along the spectral dimension across the emission lines.

The angular response of the RGS in the cross-dispersion direction was calibrated on the spectral image of the bright $\mathrm{BL}$ Lac Mkn 421, which is a point source (Brinkmann et al. 2001). Figure 4a shows the angular response to a point source in the cross-dispersion direction, overlaid on cross-dispersion emission line profiles of M 87, which shows that we do indeed resolve the core on $\gtrsim 15^{\prime \prime}$ scales.

The first two sets of profiles (in Figs. $4 a$ and b) were designed to test for the possibility that the apparent spatial intensity distributions of some of the strong emission lines are affected by radiative transfer effects. Given the typical densities and scale sizes in the cores of clusters, one estimates that the optical depth in abundant-element emission lines with oscillator strengths of order unity could be appreciable (of order 


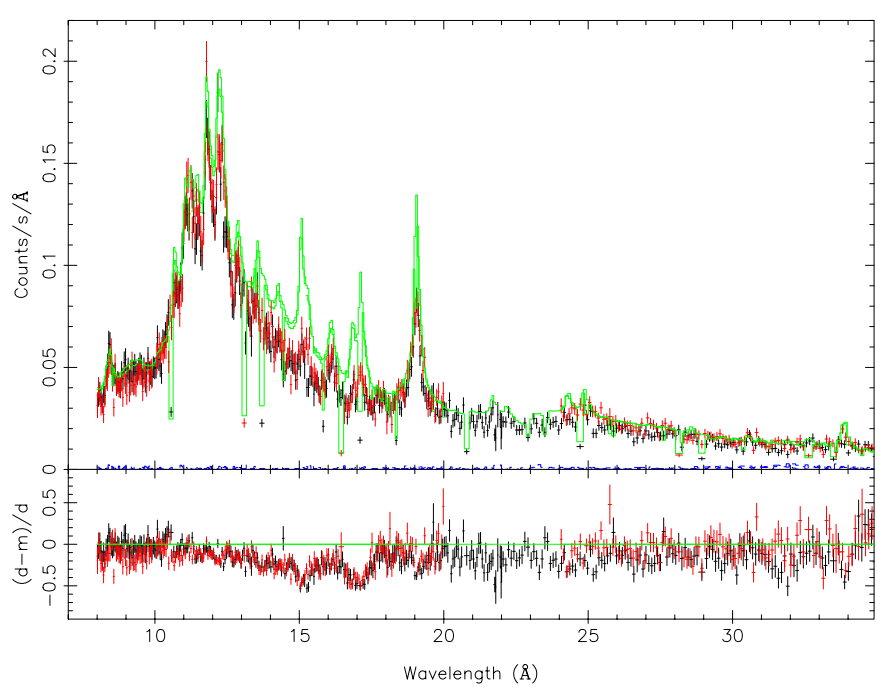

Fig. 3. The spectrum of the core of M 87: the $1 \mathrm{~T}+\mathrm{ICF}$ model is shown in green, but with all emission from the cooling flow originating from gas below $k T_{\mathrm{e}}=600 \mathrm{eV}$ included. The model over-predicts the long-wavelength range of the spectrum. It strikingly over-produces line emission from low-ionization Fe L ions, most notably Fe XVII (2p - 3d $\lambda 15.014 \AA$, $2 \mathrm{p}-3 \mathrm{~s} \lambda \lambda 17.051,17.096 \AA)$, and from O VIII (Ly $\alpha \lambda 18.97 \AA$ ).

unity), and resonance scattering of these line photons would alter their angular intensity distribution (Gilfanov et al. 1987). Understanding this effect is obviously important because it would complicate the interpretation of these intensity distributions in terms of physical gradients in temperature and abundance. In addition, constraints on individual ionic column densities, when combined with volume emission measures in the same ion, in principle over-constrain the emissivity model and allow for important consistency tests. A byproduct of such selfconsistent over-constrained models is an absolute (distanceindependent) measurement of the important physical parameters of the system. The first unambiguous detection (in the sense that it is based on single-ion spectroscopy) of this effect was recently reported by Xu et al. (2001) in the spectrum of the elliptical galaxy NGC 4636.

Figure $4 \mathrm{a}$ shows the profile of O VIII Ly $\alpha$, compared to the corresponding $\operatorname{Ly} \beta$ (the latter blended with Fe XVIII $\lambda 16.004 \AA$ ). The data indicate that the optical depth in O VIII Ly $\alpha$ is low: there is no significant difference between the $\mathrm{O}$ VIII $\operatorname{Ly} \alpha$ and $\operatorname{Ly} \beta$ profiles. It is expected that if $\operatorname{Ly} \alpha$ were optically thick, $\operatorname{Ly} \beta$ would be optically thin, due to its lower oscillator strength. Note that there is a weak trend for $\operatorname{Ly} \beta$ to appear more diffuse than $\operatorname{Ly} \alpha$, which is the contradicts our expectetions if O VIII Ly $\alpha$ were optically thick. The same figure also shows the profile for a high-oscillator strength transition in the Fe XVIII $\lambda 14.208 \AA$ doublet. Its profile is very similar to the O VIII Ly profiles.

Figure $4 \mathrm{~b}$ shows a similar set of profiles for high- and low-oscillator strength transitions in a low ionization Fe L ion, Fe XVII. The $\lambda 15.014 \AA$ transition has the highest oscillator strength of any line in the RGS band, yet its distribution looks similar to that of the low-oscillator strength $\lambda \lambda 17.051,17.096 \AA$ blend, indicating that the optical depth is small. We conclude that the core is optically thin in all important transitions.

Figure 4c displays the profile observed in the $\lambda \lambda 11.176,11.266 \AA$ blend of Fe XXIV (unresolved due to the spatial extent of the Fe XXIV emitting source), overlaid on the distribution in Fe XVII $\lambda \lambda 17.051,17.096 \AA$. The fact that the spatial distribution of the intensity in these two ions appears qualitatively similar indicates that the "multiphase" character of the gas we observe in the very core of M 87, persists on scales out to $\sim 150^{\prime \prime}$, or $\gtrsim 10 \mathrm{kpc}$.

The last comparison of profiles is between the $\operatorname{Ly} \alpha$ lines of $\mathrm{H}$-like $\mathrm{O}$ and $\mathrm{Mg}(\lambda 18.97,8.42 \AA)$, and the resonance line of He-like Si $(\lambda 6.65 \AA)$. The fact that these distributions appear similar indicates that there are no large gradients in the relative abundances of these elements with respect to each other. The O VIII Ly $\alpha$ profile appears slightly more peaked than the others, which could simply be due to the fact that the average gas temperature declines towards the very core.

\section{Summary and discussion}

We have presented high resolution soft X-ray spectroscopy of M 87. In brief, the XMM-Newton RGS spectrum shows fully spectrally resolved emission from $\mathrm{C}, \mathrm{N}, \mathrm{O}, \mathrm{Ne}, \mathrm{Mg}$, and $\mathrm{Fe}$. The angular structure of the intensity in the strong emission lines is resolved on scales of order $\gtrsim 15^{\prime \prime}$, removing much of the confusion associated with the limited spectral resolution of CCD spectrometers. We do not find any evidence for resonance scattering in the central one arcminute.

The gas in the inner $\sim 10 \mathrm{kpc}$ has a multiphase structure, as indicated by the similarity of the spatial profiles of the emission lines of Fe L ions with widely different ionization potentials. An analysis of the overall ionization balance indicates that gas at all temperatures down to $k T_{\text {min }} \approx 0.6 \mathrm{keV}$ is present, with an emission measure distribution approximately equal to that predicted for isobaric radiative cooling. Additionally, a strong approximately isothermal component of $k T_{\mathrm{e}} \approx 1.8 \mathrm{keV}$ is present, which gives rise to emission from higher Fe ionization states (e.g., Fe XXIV), whose distribution is much wider than that of the multiphased component, as seen in the CCD images (Molendi \& Pizzolato 2001; Matsushita et al. 2001). The global deposition rate of the rapidly cooling gas is about $\dot{M} \approx 6 M_{\odot} \mathrm{yr}^{-1}$ in the inner arcmin. In these respects, the core of M 87 resembles that of other cooling flow clusters.

We do not find evidence for resonance scattering redistribution in the strong emission lines. Detailed calculations of the effect of radiative transfer in M 87 (Mathews et al. 2001) predict that the line of sight average optical depth of the O VIII line is $<0.5$, consistent with our results. However, the Fe XVII lines with higher oscillator strengths are predicted to be marginally optically thick in the absence of turbulence. We do note that both our analysis and the reanalysis of the EPIC data have reduced the apparent $\mathrm{O}$ abundance deficit in the centre of $\mathrm{M} 87$, originally noted by Böhringer et al. (2001); this reduces the need for an inclusion of radiative transfer effects in the modelling of the M 87 spectrum. If anything, the accurate determination of the metal abundance depends on the level of the continuum, which in turn depends on the contribution from the 


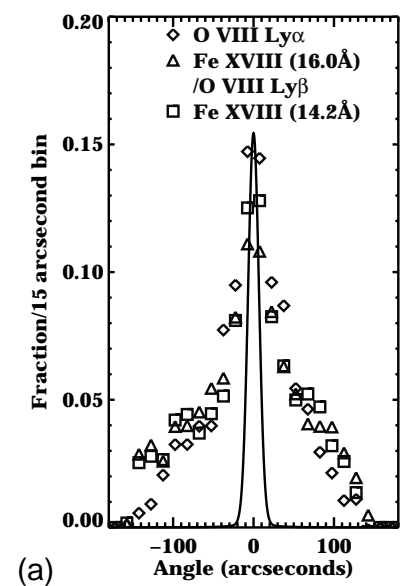

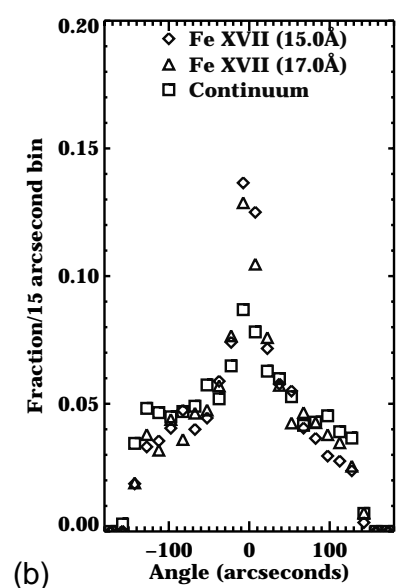

(b)

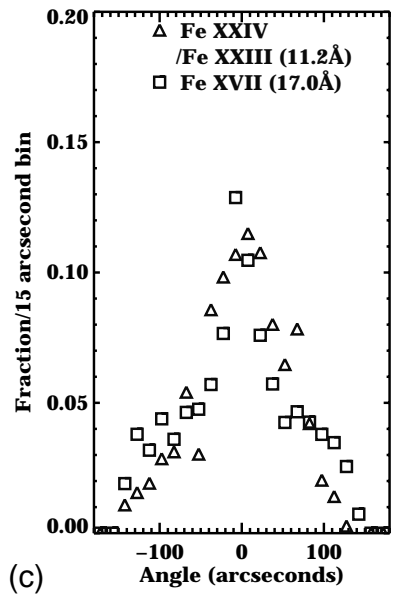

(c)

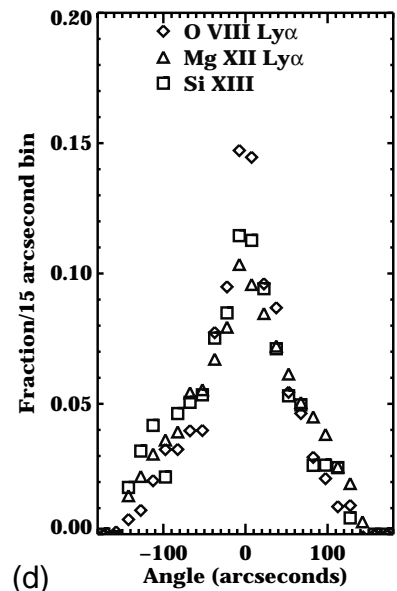

(d)

Fig. 4. Spatial intensity distribution in the cross dispersion direction of a number of diagnostic emission lines, in 15 arcsec bins. They were accumulated over $\pm 0.1 \AA$ around the wavelength of each line. The errors are smaller than the size of the symbols for the core of the distribution, and double the size of the symbols for the outer regions. The angular response of the RGS in the cross-dispersion direction is shown in a) (see Sect. 4 for more information).

active nuclear/jet emission. More confident measurements will have to wait for data from which the non-thermal emission will be subtracted, instead of modelled.

Resonance absorption scattering has been recently found in a poor cluster of galaxies (NGC 4636; Xu et al. 2001). Apart from the apparent differences between the two sources (e.g., density and temperature), both of them host a radio galaxy. The radio galaxy in NGC 4636 (Birkinshaw \& Davis 1985; Stanger \& Warwick 1986) is much weaker than that in M 87: NGC 4636 has a steep spectrum, with a core power at $2 \mathrm{~cm}$ of $6.6 \times 10^{19} \mathrm{~W} \mathrm{~Hz}^{-1}$ (Nagar et al. 2000). Both observational facts may imply that it is an old or a "dead" radio source. However, as is becoming clearer, thanks to recent numerical simulations (e.g., Brüggen et al. 2002), even "dead" radio galaxies can stir up, disrupt the intergalactic medium, and introduce turbulence. Therefore, a direct comparison between the two sources and the high resolution X-ray results, is not straightforward and more radio and $\mathrm{X}$-ray observations with high angular resolution are required to address these issues. It is expected though that turbulence in the gas will reduce the net optical depth. Such turbulence could arise as the radio jets impinge onto the intergalactic medium. We defer the detailed discussion of the relative optical depths in the different lines and the effects of turbulence to a later paper which will model such effects directly.

Our analysis of the RGS data of M 87 finds near-solar abundances of $\mathrm{C}$ and $\mathrm{N}$, $\mathrm{Ne}$ and $\mathrm{Mg}$, sub-solar for $\mathrm{O}$, and 0.8 solar for Fe. We emphasise that the absolute values for the abundances have to be regarded with some caution, because they are based on fitting a parameterised model with limited flexibility. As pointed out by many authors (e.g., Matsushita et al. 2000; Buote 2000) CCD spectra are not of sufficiently high resolution to obtain robust chemical abundances for complex thermal plasmas. In particular if there are sharp abundance gradients and multi-temperature gas the CCD data are degenerate, and several solutions can exist with different temperature and abundance values. The XMM CCD spectra for M 87 (Finoguenov et al. 2002; Gastaldello \& Molendi 2002) show both abundance gradients and complex thermal structure and therefore their interpretation is open to modelling uncertainties. Both studies use rather different models for the temperature distribution, but arrive at similar values for the abundances except for oxygen. Gastaldello \& Molendi (2002) point out that since the bulk of the emission in M 87 is locally isothermal and shows a rather smooth temperature gradient, the abundances derived from the $X M M-N e w t o n$ CCD data should not be very sensitive to the assumed fitted temperature distribution; given the high signal to noise of the XMM CCD data, all acceptable solutions should produce essentially the same distribution of emission measure with temperature.

Our thermal modelling assumptions differ from those of both Gastaldello \& Molendi (2002) and Finoguenov et al. (2002) but we do not believe that they should result in different abundances. The oxygen and $\mathrm{Fe}$ abundances we derive are the same as Finoguenov et al. (2002) and Gastaldello \& Molendi (2002) averaged over the central 1 arcmin region. However the abundances of $\mathrm{Mg}$ and $\mathrm{Ne}$, which depend on detailed modelling the Fe L shell lines in the CCD data (but not in the RGS data) are rather different with the RGS results, both being (5070) percent higher. This comparison graphically illustrates the difficulty of accurately measuring the abundances of these elements in the temperature range for which the Fe $\mathrm{L}$ shell lines are strong.

The derivation of the $\mathrm{Mg}$ abundance is of particular importance: the M 87 stellar spectra show a very high apparent $\mathrm{Mg} / \mathrm{Fe}$ ratio of $\gtrsim 2: 1$ (Terlevich \& Forbes 2002) and a supersolar $\mathrm{Mg}$ abundance which is completely incompatible with the RGS data of $\mathrm{Mg} / \mathrm{Fe} \sim 1$ and Fe less than solar. Thus, very little of the observed X-ray emitting gas can originate from stellar mass loss, contrary to the expectations of the observed stellar density and normal stellar evolutionary theory. As originally pointed out by Lowenstein \& Mathews (1991) a normal type I supernova rate combined with stellar mass loss would produce a 3-5 times solar Fe abundance in the gas. As discussed in detail by Awaki et al. (1994) the ASCA data did not detect such a high $\mathrm{Fe}$ abundance, raising the serious issue as to what has happened to the products of type I SN in the central regions of 
giant elliptical galaxies. These results enhance and deepen the mystery. The sub-solar $\mathrm{O}, \mathrm{Ne}, \mathrm{Mg}$ and $\mathrm{Fe}$ abundances are incompatible with the stellar data even if all the heavy elements in the gas originate from stellar mass loss.

We also know that M 87 is not unique. The abundance pattern in NGC 4636 (Xu et al. 2001) is extremely similar and preliminary analysis of the RGS data for another giant elliptical NGC 533 (Peterson et al. 2002) shows similar results. We thus conclude that this issue is a serious one, and it needs further attention.

The XMM-Newton RGS results pose two problems regarding the M 87 chemical abundances: i) why is the overall $\mathrm{Fe}$ abundance so low, and ii) why is the chemical composition apparently so different from the stars. We speculate that the first problem can only be solved if either the true type I SN rate is considerably reduced, or the yield of $\mathrm{Fe}$ per unit supernova is also reduced. Otherwise, Fe may be lost from the central regions of M 87 (by ram pressure stripping for example, Stevens et al. 1999), or the ISM gas is diluted due to the accretion of cluster gas, but both seem unlikely. The second issue can only be resolved if either the inferred stellar abundances are in error, or if the stellar spectra do not represent the true abundance distribution of the stars. If for example, there are two distinctive stellar populations in elliptical galaxies with different metal abundances, and the optical data register only the abundances of the metal rich group. Support for the chemical inhomogeneity of the stars in elliptical galaxies comes from deep VLT and HST observations, of NGC 5128 for example, (Harris \& Harris 2000) which shows a wide range in metallicity of the stars.

Acknowledgements. This work is based on observations obtained with $X M M-N e w t o n$, an ESA science mission with instruments and contributions directly funded by ESA Member States and the USA (NASA).

\section{References}

Anders, E., \& Grevesse, N. 1989, Geochim. Cosmochim. Acta, 53, 197

Awaki, H., Mushotzky, R., Tsuru, T., et al. 1994, PASJ, 46, L65

Belsole, E., Sauvageot, J. L., Böhringer, H., et al. 2001, A\&A, 365, L188

Birkinshaw, M., \& Davies, R. L. 1985, ApJ, 291, 32

Böhringer, H., Belsole, E., Kennea, J., et al. 2001, A\&A, 365, L181

Brinkmann, W., Sembay, S., Griffiths, G., et al. 2001, A\&A, 365, L162

Brüggen, M., Kaiser, C. R., Churazov, E., \& Enßlin, T. A. 2002, MNRAS, 331, 545

Buote, D. A. 2000, MNRAS, 311, 176

Canizares, C. R., Clark, G. W., Markert, T. H., et al. 1979, ApJ, 234, L33
Canizares, C. R., Clark, G. W., Jernigan, J. G., \& Markert, T. H. 1982, ApJ, 262, 33

den Herder, J. W., Brinkman, A. C., Kahn, S. M., et al. 2001, A\&A, 365, L7

Fabian, A. C., Nulsen, P. E. J., \& Canizares, C. R. 1984, Nature, 310, 733

Fabricant, D., Lecar, M., \& Gorenstein, P. 1980, ApJ, 241, 552

Finoguenov, A., Matsushita, K., Böhringer, H., Ikebe, Y., \& Arnaud, M. 2002, A\&A, 381, 21

Freedman, W. L., Madore, B. F., Mould, J. R., et al. 1994, Nature, 371, 757

Gastaldello, F., \& Molendi, S. 2002 [astro-ph/0202020]

Gilfanov, M. R., Sunyaev, R. A., \& Churazov, E. M. 1987, Sov. Astron. Lett., 13, 3

Harris, G. L. H., \& Harris, W. E. 2000, AJ, 120, 2423

Jansen, F., Lumb, D., Altieri, B., et al. 2001, A\&A, 365, L1

Johnstone, R. M., Fabian, A. C., Edge, A. C., \& Thomas, P. A. 1992, MNRAS, 255, 431

Kaastra, J. S., Mewe, R., \& Nieuwenhuijzen, H. 1996, in UV and $\mathrm{X}$-ray Spectroscopy of Astrophysical and Laboratory Plasmas, ed. K. Yamashita, \& T. Watanabe (Universal Academy Press, Tokyo) Kaastra, J. S., Ferrigno, C., Tamura, T., et al. 2001, A\&A, 365, L99

Lea, S. M., Mushotzky, R., \& Holt, S. S. 1982, ApJ, 262, L24

Loewenstein, M., \& Mathews, W. G. 1991, ApJ, 373, 445

Mathews, W. G., Buote, D. A., \& Brighenti, F. 2001, ApJ, 550, L31

Matsushita, K., Ohashi, T., \& Makishima, K. 2000, PASP, 52, 685

Matsushita, K., Belsole, E., Finoguenov, A., \& Böhringer, H. 2001 [astro-ph/0201242]

Molendi, S., \& Pizzolato, F. 2001, ApJ, 560, 194

Molendi, S., \& Gastaldello, F. 2002 [astro-ph/0106553]

Nagar, N. M., Falcke, H., Wilson, A. S., \& Ho, L. C. 2000, ApJ, 542, 186

Peres, C. B., Fabian, A. C., Edge, A. C., et al. 1998, MNRAS, 298, 416

Peterson, J. R., Paerels, F. B. S., kaastra, J. S., et al. 2001, A\&A, 365, L104

Peterson, J. R., et al. 2002, in preparation

Röser, H.-J., \& Meisenheimer, K. (eds.) 1999, The Radio Galaxy Messier 87, Proceedings of a workshop at Schloss Ringberg, 1997 (Springer, Heidelberg)

Stanger, V. J., \& Warwick, R. S. 1986, MNRAS, 220, 363

Stevens, I. R., Acreman, D. M., \& Ponman T. J. 1999, MNRAS, 310, 663

Stewart, G. C., Fabian, A. C., Nulsen, P. E. J., \& Canizares, C. R. 1984, ApJ, 278, 536

Tamura, T., Kaastra, J. S., Peterson, J. R., et al. 2001, A\&A, 365, L87

Terlevich, A. I., \& Forbes, D. A. 2002, MNRAS, 330, 547

Wilson, A. S., \& Yang, Y. 2001, 2002, ApJ, 568, 133

Xu, H., Kahn, S. M., Peterson, J. R., et al. 2001 [astro-ph/0110013] 